\title{
Influence of Gravitational Sympathetic Stimulation on the Surgical Plethysmographic Index
}

\author{
R. COLOMBO ${ }^{1}$, A. MARCHI ${ }^{2}$, B. BORGHI ${ }^{1}$, T. FOSSALI ${ }^{1}$, E. TOBALDINI ${ }^{3}$, S. GUZZETTI $^{4}$, \\ F. RAIMONDI ${ }^{2}$
}

${ }^{1}$ Anesthesiology and Intensive Care Unit, Azienda Ospedaliera "L. Sacco", Milano, Italy, ${ }^{2}$ Anesthesiology and Intensive Care Unit, IRCCS Istituto Clinico Humanitas, Rozzano, Italy, ${ }^{3}$ Internal Medicine 3, Azienda Ospedaliera "L. Sacco", Milano, Italy, ${ }^{4}$ Emergency Department, Azienda Ospedaliera "L. Sacco", Milano, Italy

Received April 5, 2014

Accepted July 29, 2014

On-line October 15, 2014

\section{Summary}

Surgical Plethysmographic Index (SPI), calculated from pulse photo-plethysmographic amplitude oscillations, has been proposed as a tool to measure nociception anti-nociception balance during general anesthesia, but it is affected by several confounding factor that alter the autonomic nervous system (ANS) modulation. We hypothesized that SPI may be mainly affected by sympathetic stimulation independently from nociception. We studied the effects of two sympathetic stimuli on SPI, delivered through passive head-up tilt at 45 and 90 degrees angles, in nine awake healthy adults. The sympathetic modulation was assessed by means of heart rate variability (HRV) analysis. Mean (SD) SPI significantly increased from baseline to 45 degrees [from 38.6 (13.7) to 60.8 (7.6), $\mathrm{p}<0.001)]$ and to 90 degrees angle tilt [82.3 (5.4), $\mathrm{p}<0.001]$. The electrocardiographic mean R-to-R interval significantly shortened during both passive tilts, whereas systolic arterial pressure did not change during the study protocol. HRV changed significantly during the study protocol towards a predominance of sympathetic modulation during passive tilt. Gravitational sympathetic stimulation at two increasing angles, in absence of any painful stimuli, affects SPI in awake healthy volunteers. SPI seems to reflect the sympathetic outflow directed to peripheral vessels.

\section{Key words}

Plethysmography • Sympathetic activity • Surgical pleth index • Nociception

\section{Corresponding author}

R. Colombo, Anesthesiology and Intensive Care Unit, Azienda Ospedaliera "L. Sacco", Via G.B. Grassi 74, 20157 Milano, Italy. Fax: +390239043036. E-mail: colombo.riccardo@hsacco.it

\section{Introduction}

Measuring the nociception anti-nociception balance during general anesthesia is an interesting challenge for the anesthesiologists. To date, no parameter has been definitely identified as proper marker of the adequacy of the analgesia level. The Surgical Plethysmographic Index (SPI) has been supposed to be a predictor of nociception anti-nociception balance, quite unrelated to the heart rate modulation (Ahonen et al. 2007). Indeed, SPI seems strongly related to the opiate analgesics' plasmatic concentration but quite unrelated to hypnotics' one (Huiku et al. 2007, Struys et al. 2007). SPI assumes values ranging from 0 to 100 , a high value of SPI is considered to be associated with unbalanced nociception anti-nociception with a predominance of the nociception.

SPI consists of a combined measurement of central and peripheral hemodynamic variables both under sympathetic modulation and it may be computed by the formula (Struys et al. 2007):

$$
S P I=100-\left(0.33 \cdot P B I_{\text {norm }}+0.67 \cdot P P G A_{\text {norm }}\right)
$$


where PBI and PPGA are the normalized pulse beat interval and photo-plethysmographic amplitude respectively. Normalization algorithm is a property of the manufacturer and it is yet undisclosed. Moreover SPI was found to be affected by several confounding factors including atropine administration, cardiac pacing, level of sedation, spinal anesthesia with and without sedation, and intravascular volume status (Bonhomme et al. 2011, Ilies et al. 2010, Hocker et al. 2010). We therefore hypothesized that SPI might reflect changes of sympathetic modulation independently from nociceptive stimuli. One of the most accepted maneuver exciting the autonomic cardiovascular control is head-up tilt (HUT) (Pagani et al. 1986, Montano et al. 1994, Furlan et al. 2000, Cooke et al. 1999). Tilt causes shift of blood toward lower body and reduction of the venous return. These effects induce a compensatory baroreflex-mediated increase of heart rate and peripheral vascular resistance aiming at maintain arterial pressure near to the level preceding the challenge (Robertson et al. 2012).

We conducted a study to test the hypothesis that SPI could reflect the sympathetic stimulation delivered through head up tilt in absence of nociceptive stimulation.

\section{Methods}

\section{Patients enrolment}

After local Institutional Ethical Committee approval, we studied the effects of posture change on SPI in 9 awake healthy volunteers. The study adhered to the principles of the Declaration of Helsinki for medical research involving human subjects and written informed consent was acquired from all subjects.

A sample size of 7 was needed to detect a mean (SD) SPI difference of 30 (standard deviation 15), between supine position and HUT, with a power of 0.8 and $\alpha$ error of 0.05 . All subjects were healthy, did not take any drugs, beverages containing caffeine or alcohol in the $24 \mathrm{~h}$ before the study protocol. Electrocardiogram (ECG) and photo-plethysmographic waves were recorded through a $\mathrm{S} / 5$ Advance monitor (GE, Helsinki, Finland) to a laptop computer provided with $\mathrm{S} / 5$ Collect software (GE, Helsinki, Finland). Photo-plethysmographic waves were sampled by a standard photo-plethysmographic probe, in endowment to the commercial monitor $\mathrm{S} / 5$ Advance, positioned on the third finger of the left hand. Continuous non-invasive finger blood pressures were recorded (Nexfin, BMEYE, Amsterdam, Holland) at the third finger of the right hands during the experiments. All continuous signals were sampled at $300 \mathrm{~Hz}$. The SPI values were displayed on the $\mathrm{S} / 5$ Advance monitor and they were manually recorded.

\section{Experimental protocol}

The experimental protocol consisted of a sequence of postural changes, each of them lasting $7 \mathrm{~min}$. The sequence consisted of (i) baseline recording in supine position (bas), (ii) passive head up tilt at 45 degrees angle (T1), (iii) a recovery period in supine position (R1), (iv) a passive head up tilt at 90 degrees angle (T2) and (v) a recovery in supine position (R2). The postural changes were gained with a tilt table (TILT TEST ${ }^{\circledR}$, Gardhen Bilance, Pomigliano d'Arco, Italy). During the study sequence the healthy volunteers were asked to lie calmly, breathing in rhythm with a metronome at 18 breaths per minute $(0.3 \mathrm{~Hz})$.

\section{Autonomic nervous system assessment}

At each study phase, the autonomic nervous system modulation was assessed by means of the heart rate variability (HRV) analysis according to the recommendations of the European Society of Cardiology Task Force (Task Force of the European Society of Cardiology and the North American Society of Pacing and Electrophysiology 1996). R-to-R interval between consecutive beats was detected on the ECG using a semiautomatic derivative/threshold method (Porta et al. 1998); the tachogram was extracted and reviewed by an investigator to avoid erroneous detections or missed beats. If isolated ectopic beats occurred, these were removed and linearly interpolated using the closest values unaffected by ectopic beats. Sequences of 300 consecutive heart beats inside each experimental phase were analyzed according to an autoregressive model (Pagani et al. 1986). The mean and the variance of heart period are expressed in ms and $\mathrm{ms}^{2}$ respectively. The power spectral density was factorized into components each of them characterized by a central frequency. A spectral component was labeled as LF if its central frequency was between 0.04 and $0.15 \mathrm{~Hz}$, while it was classified as HF if its central frequency was between 0.15 and $0.5 \mathrm{~Hz}$ (Task Force of the European Society of Cardiology and the North American Society of Pacing and Electrophysiology 1996). The LF and HF powers were defined as the sum of the powers of all LF and HF spectral components respectively. The HF power was considered to represent respiration-driven vagal modulation of heart rate (Akselrod et al. 1981, Pagani et al. 1986). There is more disagreement about the low 
frequency oscillation of heart rate. LF may reflect vasomotor activity, which is an indirect index of sympathetic nerve activity, as well as partially affected by parasympathetic activity (Hopf et al. 1995, Goldsmith et al. 1992, Cook et al. 1991). To rule out the effect of changes of total power spectrum densities on LF and HF components, spectral values were expressed in normalized units (NU). Normalization consisted in dividing the power of a given spectral component by the total power minus the power below $0.04 \mathrm{~Hz}$ (Very Low Frequency spectral component), and multiplying the ratio by 100 . The ratio of the LF power to the HF (LF/HF) was considered a marker of the balance between sympathetic and vagal modulation directed to the heart (Pagani et al. 1986).

The investigator (AM) who analyzed the heart variability data was blinded to the SPI value.

\section{Statistical analysis}

For continuous variables, we used mean (SD), or median (IQR) when not normally distributed. The normality distribution was checked using the KolmogorovSmirnov test. We performed one way analysis of variance (ANOVA) for repeated measures followed by post-hoc Tukey's test for multiple comparison. Statistical analysis was carried out using a commercial statistical software (GraphPad Prism 5 for Windows). A $\mathrm{p}<0.05$ was considered as significant for two tails tests.

\section{Results}

The SPI trends recorded from the nine volunteers [mean age 26.7 (4) years, F to $M$ ratio 4/5] are shown in the Fig. 1. The postural changes modified significantly the SPI and mean R-to-R interval (ANOVA $\mathrm{p}<0.001$ ), while systolic arterial pressure remained unchanged. SPI increased significantly from baseline to T1 (Table 1). The increase was higher at T2 (82.3 [5.4], $\mathrm{p}<0.001$ vs. baseline). After each head up tilt the mean SPI decreased significantly at a value below the baseline value, respectively 24.3 (8.5) at $\mathrm{R} 1$ and 17.9 (4.4), $\mathrm{p}<0.001$ at $\mathrm{R} 2$. The electrocardiographic R-to-R interval decreased significantly during each head up tilt with respect to the basal values and increased during $\mathrm{R} 1$ and R2 (ANOVA $\mathrm{p}<0.001)$. The electrocardiographic mean R-to- $R$ interval shortened from baseline to $T 1 \quad(p<0.05)$ and T2 $(\mathrm{p}<0.001)$. During both tilts at 45 degrees, the high frequency spectral density component of the heart rate variability $\left(\mathrm{HF}_{\mathrm{NU}}\right)$ showed a trend towards a reduction [36.7 (26.5) NU at T1 vs. 49.5 (17.2) NU at baseline] and it was significantly lower during head up tilt at 90 degrees [19.5 (11.6) NU, $\mathrm{p}<0.001$ vs. baseline] (Fig. 2A). Furthermore, during tilt, LF/HF ratio increased toward a predominance of sympathetic modulation over the vagal one [5.6 (3.3) at T2 vs. $1.2(0.6)$ at baseline, $\mathrm{p}<0.0001]$ (Fig. 2B).

\section{Discussion}

Our study has shown that sympathetic orthostatic stimuli affect significantly the SPI in healthy awake volunteers. Furthermore, the changes are more marked at high tilt angle.

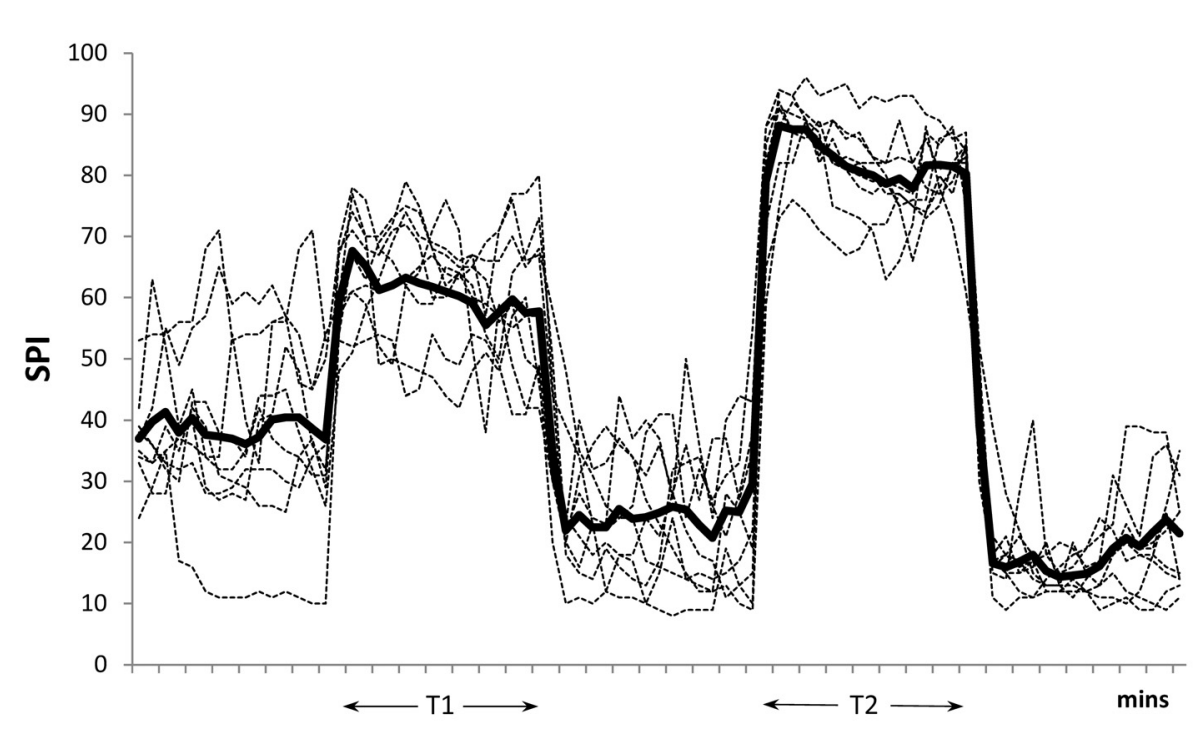

Fig. 1. Thin lines represent Surgical Pleth Index (SPI) trends recorded from the nine volunteers, thick line represents the mean value of SPI from all subjects. $T 1$, tilt at 45 degrees; $T 2$, tilt at 90 degrees 
The pulse plethysmographic amplitude is due to pulsatile changes of the arteriolar blood volume into the tissue. The blood volume pulsations $(\Delta \mathrm{V})$ are related both to the systemic intravascular pulse pressure $(\Delta \mathrm{P})$ and to the distensibility of the vascular wall (D) (Burton 1954) following the relationship: $\Delta V=\Delta P \bullet D$. The distensibility is influenced by intravascular volume status and by sympathetic-mediated vasoconstrictor tone at the peripheral arterioles, whereas vagal fibers are absent in the peripheral vessels. As a consequence SPI is strongly affected by any stimulus, not only nociceptive ones, exciting the sympathetic branch of the autonomic nervous system.

Table 1. Hemodynamics, Surgical Pleth Index and heart rate variability during study phases.

\begin{tabular}{|c|c|c|c|c|c|c|}
\hline & Baseline & T1 & $\mathbf{R} 1$ & $\mathbf{T} 2$ & $\mathbf{R 2}$ & $\begin{array}{c}P \\
(\text { ANOVA) }\end{array}$ \\
\hline RRmean (ms) & $921(148)$ & $830(79) \dagger \#$ & $1004(82)^{* \pi}$ & $731(74) \dagger+$ & 995 (90) & $<0.0001$ \\
\hline $\mathrm{SAP}(\mathrm{mm} \mathrm{Hg})$ & $129(11)$ & $126(9)$ & $130(10)$ & $130(11)$ & $129(15)$ & 0.64 \\
\hline$S P I$ & $38.6(13.7)$ & $60.8(7.6) \dagger \#$ & $24.3(8.5)^{*} \S$ & $82.3(5.4) \dagger+$ & $17.9(4.4) \dagger$ & $<0.0001$ \\
\hline$P S D_{R R}\left(m s^{2}\right)$ & $2833(952)$ & $3201(2317)$ & $4452(2624)$ & $2648(1774)$ & $4525(3661)$ & 0.02 \\
\hline$L F_{R R}(N U)$ & $49.1(17)$ & $67.3(14.2)$ & $51.3(17.9) \S$ & $78.7(12.1) \dagger+$ & $46.2(14.6)$ & $<0.0001$ \\
\hline
\end{tabular}

* $p<0.05$ vs. baseline; $+p<0.001$ vs. baseline; $\# p<0.001$ vs. R1, T2 and R2; $\S p<0.001$ vs. T2; $\neq p<0.001$ vs. R2; $9 \mathrm{p}<0.01$ vs. T1, T2 and R2 (Tukey's post-hoc test). RRmean, mean R-to-R interval; SAP, systolic arterial pressure; SPI, Surgical Pleth Index; $P_{\text {SDR }}$, total power spectral density of the R-to-R variance; $L_{R R}$, low frequency spectral band or R-to-R intervals in normalized units; $T 1$, head-up tilt at 45 degrees; R1, recovery after $\mathrm{T} 1 ; \mathrm{T} 2$, head-up at 90 degrees; R2, recovery after $\mathrm{T} 2$

A

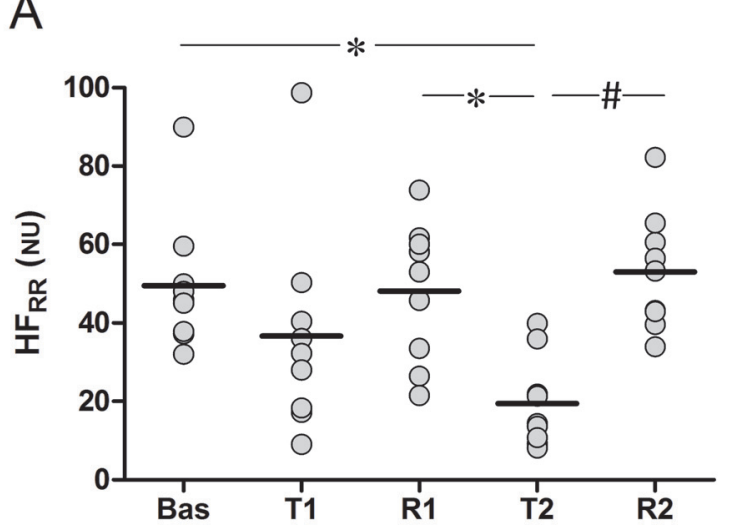

B

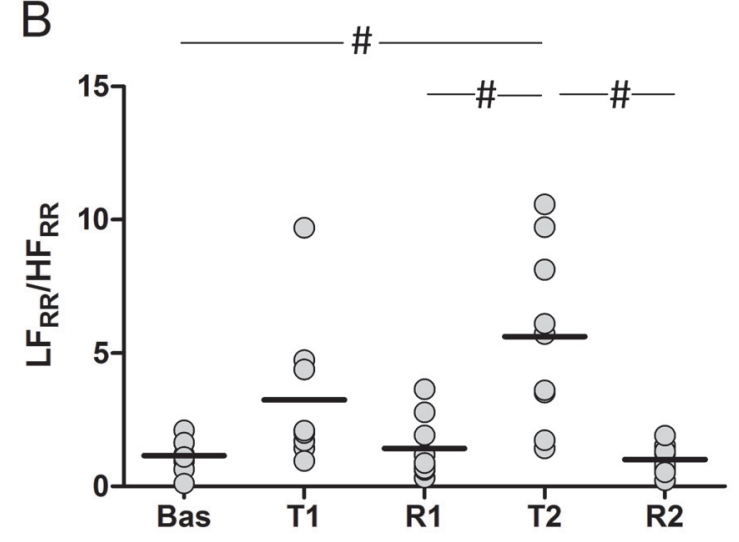

Fig. 2. Heart rate variability during the study protocol. Panel $\boldsymbol{A}$, High frequency spectral density of R-to-R intervals expressed in normalized units (NU). Panel $\boldsymbol{B}$, Low frequency to High frequency ratio of heart rate variability spectral density $\left(\mathrm{LF}_{\mathrm{RR}} / \mathrm{HF}_{\mathrm{RR}}\right)$ as a marker of sympatho-vagal balance. Both HF and LF/HF showed a significant overall variation (ANOVA $p<0.0001$ ). $* p<0.001, \# p<0.0001$ between the specified study phases (Tukey's post-hoc test). Bas, baseline; T1, head-up tilt at 45 degrees; R1, recovery after T1; T2, head-up at 90 degrees; R2, recovery after T2

During orthostatic position, gravitational fluid shift toward the lower part of the body would cause severe orthostatic hypotension if not counteracted by compensatory mechanisms mediated by baroreflex activation of sympathetic outflow. There are evidences suggesting that vasoconstrictor capability is a contributor to orthostatic tolerance in humans (Fu et al. 2004). The head-up tilt has been shown to cause hormonal changes: plasma renin activity, aldosterone, adrenocorticotropic hormone (ACTH), adrenomedullin and vasopressin increase significantly (Smith et al. 1994, HinghoferSzalkay et al. 2011). Moreover the plasma vasopressin was found to increase significantly, mediated by deloading of carotid baroreceptors induced by the acute fall in hydrostatic pressure at the level of the carotid sinus (Kamegai et al. 1992). Although we did not collect blood samples in order to measure plasma concentration of hormones, we suppose that the prompt increase and 
decrease of SPI were due principally to neurally mediated vasoconstriction. During tilt, both at low and high degrees, the R-R interval decreased while SAP remained unchanged as it is expected in physiological conditions with the activation of the baroreflex response. The variations of SPI were quick, reaching a new steady level in less than a minute, thus it seems unlikely that these variations were due to hormonal changes. Many earlier human studies have reported that increasing the tilt angle of HUT results in greater muscle sympathetic nerve activity (MSNA) response, indicating the amplitude dependence of sympathetic activation in response to orthostatic stress. One of the important findings is that stronger orthostatic stress results in greater MSNA response during incremental HUT (Cooke et al. 1999, Fu et al. 2009, Iwase et al. 1991, Kamiya et al. 2000). These results demonstrated that MSNA plays an important role in hemodynamic regulation during incremental changes in posture indicating the amplitude dependence of orthostatic MSNA activation.

We found that SPI reduced below to the basal value during recovery from head-up position. It may be explained with sudden increase in venous return, in central blood volume and stretch of atrial and pulmonary mechanoceptors triggering vagal outflow and inhibiting sympathetic modulation at a greater extent (Crystal and Salem 2012). During HUT, the vascular resistance has been demonstrated to increase in the upper limb by $85 \%$ (Kitano et al. 2005) but it increased more markedly in supine position with applied lower-body negative pressure, suggesting a different role of cardiopulmonary mechanoceptors or baroceptors activation. Moreover, forearm blood flow is well demonstrated to increase during small angles head-down tilt. This increase the afferent firing rate by the cardiopulmonary receptors and probably inhibits sympathetic outflow in the central nervous system (Nagaya et al. 1995). Like this, we suppose that the quick shift from head-up position to supine position at zero degrees causes a pooling of blood to the cardiac chambers and to the lungs mimicking a head-down tilt. Finally, the forearm blood flow is the principal determinant of the PPGA.

Ilies et al. (2012) studied SPI during $30^{\circ}$ headdown and $30^{\circ}$ head-up in lithotomy position in patients undergoing general anesthesia, spinal anesthesia, spinal anesthesia with sedation and in awake volunteers. They found that putting patients in the lithotomy position significantly decreases the SPI, while $30^{\circ}$ HUT increases it. However drugs (hypnotics and opiates) used during general anesthesia and the subarachnoid block have a marked effects on vasoconstrictor sympathetic tone. Moreover the lithotomy position (with $90^{\circ}$ flexion at knees and ankles) may cause a blood shift toward the trunk and cardiac chambers that alters the hemodynamic cardiac response. We applied a standard sympathetic stimulus with two angles HUT and studied its effects on SPI, to minimize these confounders.

Our study has few limitations. We applied two large angles HUT that are not used routinely during general anesthesia. As a result, it may make difficult to generalize our findings. However we tested the hypothesis that a well standardized sympathetic stimulus, not orthostatic position per se, could affect SPI. Moreover it was demonstrated that vestibular otolith stimulation increases MSNA and have an additive effect on baroreflex in humans (Ray 2000). We cannot discriminate the baroreflex and otolith stimulation effects on SPI. Moreover we cannot argue about the drug effects on baroreflex-vestibular interaction during general anesthesia.

In conclusion we found that gravitational sympathetic stimuli at two different angles affect proportionally the SPI in awake healthy volunteers. The results of this study suggest that SPI reflects the sympathetic modulation directed to the vessels through sympathetic mediated vasoconstriction in response to a multitude of afferences, not only nociception. It should be taken in account in interpreting the displayed SPI value in a clinical setting.

\section{Conflict of Interest}

There is no conflict of interest.

\section{References}

AHONEN J, JOKELA R, UUTELA K, HUIKU M: Surgical stress index reflects surgical stress in gynaecological laparoscopic day-case surgery. Br J Anaesth 98: 456-461, 2007.

AKSELROD S, GORDON D, UBEL FA, SHANNON DC, BERGER AC, COHEN RJ: Power spectrum analysis of heart rate fluctuation: a quantitative probe of beat-to-beat cardiovascular control. Science 213: 220-222, 1981. 
BONHOMME V, UUTELA K, HANS G, MAQUOI I, BORN JD, BRICHANT JF, LAMY M, HANS P: Comparison of the surgical Pleth Index with haemodynamic variables to assess nociception-anti-nociception balance during general anaesthesia. Br J Anaesth 106: 101-111, 2011.

BURTON AC: Relation of structure to function of the tissues of the wall of blood vessels. Physiol Rev 34: 619-642, 1954.

COOK JR, BIGGER JT, KLEIGER RE, FLEISS JL, STEINMAN RC, ROLNITZKY LM: Effect of atenolol and diltiazem on heart period variability in normal persons. $J$ Am Coll Cardiol 17: 480-484, 1991.

COOKE WH, HOAG JB, CROSSMAN AA, KUUSELA TA, TAHVANAINEN KU, ECKBERG DL: Human responses to upright tilt: a window on central autonomic integration. J Physiol 517: 617-628, 1999.

CRYSTAL GJ, SALEM MR: The Bainbridge and the "reverse" Bainbridge reflexes: history, physiology, and clinical relevance. Anesth Analg 114: 520-532, 2012.

FU Q, WITKOWSKI S, LEVINE BD: Vasoconstrictor reserve and sympathetic neural control of orthostasis. Circulation 110: 2931-2937, 2004.

FU Q, OKAZAKI K, SHIBATA S, SHOOK RP, VANGUNDAY TB, GALBREATH MM, REELICK MF, LEVINE BD: Menstrual cycle effects on sympathetic neural responses to upright tilt. J Physiol 587: 2019-2031, 2009.

FURLAN R, PORTA A, COSTA F, TANK J, BAKER L, SCHIAVI R, ROBERTSON D, MALLIANI A, MOSQUEDA-GARCIA R: Oscillatory patterns in sympathetic neural discharge and cardiovascular variables during orthostatic stimulus. Circulation 101: 886-892, 2000.

GOLDSMITH RL, BIGGER JT, STEINMAN RC, FLEISS JL: Comparison of 24-hour parasympathetic activity in endurance-trained and untrained young men. $J$ Am Coll Cardiol 20: 552-558, 1992.

HINGHOFER-SZALKAY H, LACKNER HK, ROSSLER A, NARATH B, JANTSCHER A, GOSWAMI N: Hormonal and plasma volume changes after presyncope. Eur J Clin Invest 41: 1180-1185, 2011.

HOCKER J, BROCH O, GRASNER JT, GRUENEWALD M, ILIES C, STEINFATH M, BEIN B: Surgical stress index in response to pacemaker stimulation or atropine. Br J Anaesth 105: 150-154, 2010.

HOPF HB, SKYSCHALLY A, HEUSCH G, PETERS J: Low-frequency spectral power of heart rate variability is not a specific marker of cardiac sympathetic modulation. Anesthesiology 82: 609-619, 1995.

HUIKU M, UUTELA K, VAN GILS M, KORHONEN I, KYMALAINEN M, MERILAINEN P, PALOHEIMO M, RANTANEN M, TAKALA P, VIERTIO-OJA H, YLI-HANKALA A: Assessment of surgical stress during general anaesthesia. Br J Anaesth 98: 447-455, 2007.

ILIES C, GRUENEWALD M, LUDWIGS J, THEE C, HOCKER J, HANSS R, STEINFATH M, BEIN B: Evaluation of the surgical stress index during spinal and general anaesthesia. Br J Anaesth 105: 533-537, 2010.

ILIES C, LUDWIGS J, GRUENEWALD M, THEE C, HANF J, HANSS R, STEINFATH M, BEIN B: The effect of posture and anaesthetic technique on the surgical pleth index. Anaesthesia 67: 508-513, 2012.

IWASE S, MANO T, WATANABE T, SAITO M, KOBAYASHI F: Age-related changes of sympathetic outflow to muscles in humans. J Gerontol 46: M1-M5, 1991.

KAMEGAI M, KRISTENSEN MS, WARBERG J, NORSK P: Carotid baroreflexes and plasma vasopressin in humans during head-up tilt. Am J Physiol 263: R318-R323, 1992.

KAMIYA A, IWASE S, SUGIYAMA Y, MANO T, SUDOH M: Vasomotor sympathetic nerve activity in men during bed rest and on orthostasis after bed rest. Aviat Space Environ Med 71: 142-149, 2000.

KITANO A, SHOEMAKER JK, ICHINOSE M, WADA H, NISHIYASU T: Comparison of cardiovascular responses between lower body negative pressure and head-up tilt. J Appl Physiol 98: 2081-2086, 2005.

MONTANO N, RUSCONE TG, PORTA A, LOMBARDI F, PAGANI M, MALLIANI A: Power spectrum analysis of heart rate variability to assess the changes in sympathovagal balance during graded orthostatic tilt. Circulation 90: 1826-1831, 1994.

NAGAYA K, WADA F, NAKAMITSU S, SAGAWA S, SHIRAKI K: Responses of the circulatory system and muscle sympathetic nerve activity to head-down tilt in humans. Am J Physiol 268: R1289-R1294, 1995.

PAGANI M, LOMBARDI F, GUZZETTI S, RIMOLDI O, FURLAN R, PIZZINELLI P, SANDRONE G, MALFATTO G, DELl'ORTO S, PICCALUGA E, TURIEL M, BASELli G, CERUTTI S, MALLIANI A: Power spectral analysis of heart rate and arterial pressure variabilities as a marker of sympatho-vagal interaction in man and conscious dog. Circ Res 59: 178-193, 1986. 
PORTA A, BASELLI G, CAIANI E, MALLIANI A, LOMBARDI F, CERUTTI S: Quantifying electrocardiogram RTRR variability interactions. Med Biol Eng Comput 36: 27-34, 1998.

RAY CA: Interaction of the vestibular system and baroreflexes on sympathetic nerve activity in humans. Am J Physiol 279: $\mathrm{H} 2399-\mathrm{H} 2404,2000$.

ROBERTSON D, DIEDRICH A, CHAPLEAU MW: Editorial on arterial baroreflex issue. Auton Neurosci 172: 1-3, 2012.

SMITH JJ, PORTH CM, ERICKSON M: Hemodynamic response to the upright posture. J Clin Pharmacol 34: 375386, 1994.

STRUYS MM, VANPETEGHEM C, HUIKU M, UUTELA K, BLYAERT NB, MORTIER EP: Changes in a surgical stress index in response to standardized pain stimuli during propofol-remifentanil infusion. Br J Anaesth 99: 359-367, 2007.

TASK FORCE OF THE EUROPEAN SOCIETY OF CARDIOLOGY AND THE NORTH AMERICAN SOCIETY OF PACING AND ELECTROPHYSIOLOGY: Heart rate variability: standards of measurement, physiological interpretation and clinical use. Circulation 93: 1043-1065, 1996. 